\title{
External auditory canal atresia-vertical talus-hypertelorism syndrome
}

INSERM

\section{Source}

INSERM. (1999). Orphanet: an online rare disease and orphan drug data base. External auditory canal atresia-vertical talus-hypertelorism syndrome. ORPHA:3023

A rare, genetic, multiple cong enital anomalies/dysmorphic syndrome characterized by the triad: congenital, bilateral, symmetrical, subtotal, external auditory canal atresia, bilateral vertical talus and increased interocular distance. 